\title{
Investigating Coupling Strength in Multi-agent System
}

\author{
Mingyong Liu, Panpan Yang and Xiaokang Lei \\ School of Marine Science and Technology, Northwestern Polytechnical University, \\ $X i$ 'an 710072, China \\ liumingyong@nwpu.edu.cn,yangpanpan1985@126.com,ray_com@163.com
}

\begin{abstract}
Coupling strength represents the interaction intensity between individuals, which is likely to affect both the convergence and stability properties of multi-agent system. In this paper, a coupling strength based approach is proposed to study the internal interaction mechanisms of multi-agent system. Inspired by the interaction behaviors of biological flocks, three factors, including position, velocity and number of neighbors, are first proposed to generate its relationship with interaction intensity. Then, coupling strength is analytically constructed by synthesizing these factors. Finally, two different cases, with both fixed and varying coupling strength, are simulated to illustrate the effectiveness of the proposed coupling strength in flocking control applications. Moreover, the effects of each factor on flocking are extensively studied.
\end{abstract}

Keywords: Multi-agent system, coupling strength, local interaction, flocking

\section{Introduction}

Multi-agent system has received considerable attention in the past few years due to its potential applications in the distributed sensing of mobile sensor networks, formation keeping of unmanned aerial vehicles and cooperative working of autonomous robot teams [1]. Generally, the above issues all demand the whole group of agents working cooperatively through a distributed protocol. Therefore, research on those distributed protocols, which are often called interaction rules, was extensively performed by biologist, physicist, and even computer scientist. Various interaction frameworks were proposed to explain the underlying interaction mechanisms of flocking [7-11]. In addition, researchers from mathematics, physics, control science and computer technology also showed great interest in this field and presented diverse interaction rules to reproduce the collective behavior based on the platform of multi-agent system [2-4, 12-16].

As far as we know, most of the aforementioned work utilized topological connectivity to denote the relationship or coupling strength between two agents. As is shown in Figure 1 (a), if agent $i$ and agent $j$ are connected, their coupling strength $c_{i j}$ is defined as 1 , otherwise, $c_{i j}=0$. However, this simplification may not well match the peculiarities of multi-agent system as multi-agent system is a dynamically evolving system in which the relationships between different agents are changing over time. Thus, coupling strength between agents may vary from one pair to another, depending on the relative distance of agents or other factors [14]. In addition, coupling strength would also influence the convergence and stability of multi-agent system, e.g., inappropriate coupling strength may cause divergence, oscillation or even other unexpected behaviors [15] Therefore, study on coupling strength is of great significance in both theory and application.

Compared with the existing works on interaction rules, coupling strength between different agents has not been extensively explored. Some preliminary works related to coupling strength can be found in [14-16]. As is shown in Figure 1 (b), the authors used asymmetric coupling matrix $W=\left[\omega_{i j}\right] \in \square^{n \times n}$ to represent the relationship between agents, 
where $\omega_{i j}$ is the weighted coupling strength and can be different from each other. However, such weighted values remain constant during the evolution of a multi-agent system. A fixed set of weighted values does not accurately reflect the real working conditions of multi-agent system. Moreover, Olfati-Saber utilized position dependent adjacency matrix $A(q)=\left[a_{i j}\right] \in \square^{n \times n}$ to denote the varying coupling strength according to the topology evolution of a multi-agent system [4], but other factors that are relevant to coupling strength still remain unknown.

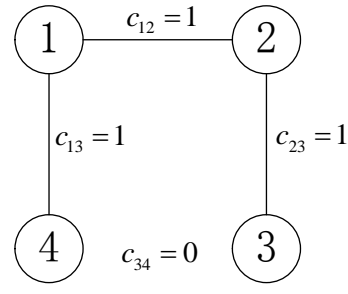

(a)

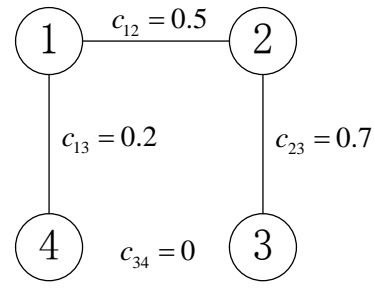

(b)

Figure 1. Interaction Intensity between Individuals

The properties of multi-agent system are akin to that of biological systems. Coupling strength between individuals is of significant influence on natural flocks, for example, the non-uniform coupling strength in prey-predator interactions plays a crucial role in the food web dynamics [17]. Bees appear to have tighter coupling strength with fast flying bees [18]. Those differences and variations in coupling strength are of vital significance in animal's foraging, migration and surviving, which also provides new inspirations for the research of coupling strength in multi-agent system.

In this paper, we tend to investigate the coupling strength between agents according to different relevant factors. Inspired by the mutual interaction behavior between individuals in biological flocks, three factors, including position, velocity and number of neighbors, are proposed. By synthesizing these factors, we obtain the exact form of coupling strength and apply it to the flocking control of multi-agent system. Comparative study demonstrates its advantage in enhancing the performance of flocking. Furthermore, extended simulation studies on the relationship between coupling strength and different factors are implemented.

The organization of this paper is as follows. The multi-agent system model and some definitions are provided in Section 1. In Section 2, the coupling strength between agents is investigated and formulated. In Section 3, comparative simulation studies and extended discussions are performed. Section 4 offers the concluding remarks of this paper.

\section{Problem Formulation}

Consider a multi-agent system consisting of $N$ mobile agents which are moving in a two-dimensional Euclidean space with the following double integrator dynamics:

$$
\left\{\begin{array}{l}
\dot{p}_{i}=\dot{v}_{i} \\
\dot{v}_{i}=u_{i}, i=1,2, \cdots, N
\end{array}\right.
$$

where $p_{i} \in \square^{2 \times 2}$ is the position vector of agent $i, v_{i} \in \square^{2 \times 2}$ is its velocity vector and $u_{i} \in \square^{2 \times 2}$ is the control input acting on it.

In the flocking control of multi-agent system, one needs to design the control input $u_{i}$ such that the whole group can move according to the control requirements. For each agent, the following assumptions are made:

(1) Agents are identical ones. They execute the same rule but act independently.

(2) Agents have limited perception capability and only have local information. 
(3) The motion of each agent is only determined by the agents within its perception range.

After the formulation of the mathematical model of multi-agent system, several basic concepts are defined as below:

Definition 1 Neighbor: Agents that are within the perception range $R$ of agent $i$ are called the neighbors of $i$, which is defined as a neighbor set $N_{i}=\left\{j:\left\|p_{j}-p_{i}\right\| \leq R, j \neq i, j=1,2, \cdots, N\right\}$, where $\left\|p_{j}-p_{i}\right\|=\sqrt{\left(p_{j}-p_{i}\right)^{\mathrm{T}}\left(p_{j}-p_{i}\right)}$ is the Euclidean distance between agent $i$ and $j$.

Definition 2 Adjacency Matrix: From the perspective of graph theory, we use adjacency matrix $A=\left[a_{i j}\right] \in \square^{N \times N}$ to denote the neighbor relationship of individuals, where

$$
a_{i j}= \begin{cases}1, & j \in N_{i} \\ 0, & j \notin N_{i}\end{cases}
$$

Here, $a_{i j}$ also indicates the connectivity of agents. If $a_{i j}=1$, agent $i$ and $j$ are connected, otherwise they are disconnected and there is no information flow between the two agents.

Definition 3 Heading: The moving direction of agent $i$ is defined as its heading $\theta_{i}$, which is written as

$$
\theta_{i}=\arctan \frac{v_{i y}}{v_{i x}}
$$

where $v_{i x}$ and $v_{i y}$ are the velocity of agent $i$ in $x$ and $y$ axis, respectively.

Definition 4 Coupling Strength: The interaction intensity between agents is defined as the coupling strength, which describes the internal interaction dynamics of multi-agent system.

For a multi-agent system, coupling strength can be illustrated in the following matrix form:

$$
C=\left[\begin{array}{cccc}
0 & c_{12} & \cdots & c_{1 N} \\
c_{21} & 0 & \cdots & c_{2 N} \\
\vdots & \vdots & \vdots & \vdots \\
c_{N 1} & c_{N 2} & \cdots & 0
\end{array}\right] \in \square^{N \times N}
$$

where $c_{i j}=f\left(p_{i j}, v_{i j},\left|N_{i}\right|, \cdots\right)$ is the coupling strength function relevant to many factors like position, velocity, number of neighbors and etc. The relative position and velocity between agent $i$ and $j$ are denoted by $p_{i j}$ and $v_{i j}$, respectively. In addition, the number of neighbors of agent $i$ is denoted by $\left|N_{i}\right|$.

The main purpose of this paper is to explore the relevant factors of $c_{i j}$, formulate its detailed mathematical form and investigate its effect on the flocking control of multiagent system.

\section{Coupling Strength Formulation between Agents}

In this section, according to the research results of biological flocks, three factors, including position, velocity and number of neighbors are proposed to describe the coupling strength between agents and the detailed form of each factor is formulated mathematically.

\subsection{Position Factor}

In multi-agent system, issues related to position are of higher importance. This can be seen in particular in bird flocks, where the movement of each bird is mostly influenced by its nearest neighbors $[19,20]$. Accordingly, the relative distance between different agents 
is the most significant factor of coupling strength. For this reason, we propose a position dependent coupling term as follows:

$$
\zeta_{i j}=\frac{\rho_{h}(z)}{r_{i j}}
$$

where $r_{i j}>0$ is the coefficient of position coupling strength, $\rho_{h}(z)$ is a smooth bump function that varies between 0 and 1 , its exact form is

$$
\rho_{h}(z)= \begin{cases}1, & z \in[0, h) \\ \frac{1}{2}\left[1+\cos \left(\pi \frac{z-h}{1-h}\right)\right], & {[h, 1]} \\ 0, & \text { others }\end{cases}
$$

where $h \in(0,1)$ is the threshold parameter, $z$ is the position coupling ratio

$$
z=\frac{\sqrt{1+\left\|p_{j}-p_{i}\right\|^{2} / \varepsilon}-1}{\sqrt{1+R^{2} / \varepsilon}-1}
$$

where $\varepsilon>0$ is the adjusting parameter.

\subsection{Velocity Factor}

Apart from position, velocity is another important factor to be considered. In some occasions, velocity variation can be more attractive as individuals are very sensitive to fast moving neighbors in honeybee swarms, bird flocks and even human crowds [21]. For example, uninformed bees in honeybee swarm appear to have tighter relationship with streaker bees [18].

Inspired by such natural phenomenon, we formulate the velocity related coupling term $\omega_{i j}$ as

$$
\dot{\omega}_{i j}=\mu_{i j} a_{i j}\left(v_{i}-v_{j}\right)^{\mathrm{T}}\left(v_{i}-v_{j}\right)
$$

where $\mu_{i j}>0$ is the coefficient of velocity coupling strength.

\subsection{Neighbor Factor}

Given that the movement of agents in a multi-agent system is determined by the motion of its neighbors, the number of neighbors is the next factor to be taken into account. Note that the more neighbors any agent has, the less it will be influenced by a single neighbor [22]. Therefore, we add neighbor coupling term to coupling strength and its general form is as follows:

$$
\eta_{i j}= \begin{cases}a_{i j} \frac{\kappa_{i j}}{\left|N_{i}\right|^{\beta}}, & \left|N_{i}\right| \neq 0 \\ 0, & \left|N_{i}\right|=0\end{cases}
$$

where $\kappa_{i j}>0$ is the coefficient of neighbor coupling strength, $\left|N_{i}\right|$ is the number of neighbors of agent $i$, and $\beta \in N^{+}$is a tunable parameter.

As for a non-isolated agent, $\left|N_{i}\right|>0$ is a positive integer. Thus, for any $\kappa_{i j}$ of the neighbor of agent $i$, we have $\eta_{i j}<\kappa_{i j}$, which demonstrates that the introduction of neighbor coupling strength $\eta_{i j}$ will weaken the relationship with a specific neighbor. 


\subsection{Coupling Strength}

The construction of coupling strength is a fusion process of the above three factors. According to the characteristics of each factor, we synthesize the above factors and obtain the explicit form of coupling strength as

$$
c_{i j}=\left(\zeta_{i j}+\omega_{i j}\right) \eta_{i j}
$$

From equation (10), it can be seen that coupling strength is a combination of the position, velocity and neighbor information that may influence the interaction intensity between agents. We can also see that $c_{i j}$ is proportional to the relative velocity between two agents and inverse to the relative position and number of neighbors, which is consistent with the mutual interaction phenomena observed in natural flocks and can be used to indicate the interaction intensity between agents in the flock.

Moreover, we adopt three tunable coefficients $r_{i j}, \mu_{i j}$ and $\kappa_{i j}$ to adjust the magnitude of $\zeta_{i j}, \omega_{i j}$ and $\eta_{i j}$ in different circumstances.

\section{Simulation and Discussions}

In order to investigate the effect of the proposed coupling strength on the flocking control of multi-agent system, a comparative study is performed with two simulation cases: flocking with $0 / 1$ fixed coupling strength and flocking with varying coupling strength designed in equation (10).

\subsection{Flocking Control Law}

For a multi-agent system with dynamics (1), the flocking control input $u_{i}$ is designed as

$$
u_{i}=-\sum_{j \in N_{i}} \nabla_{p_{i j}} V_{i j}-\sum_{j \in N_{i}} c_{i j}\left(v_{i}-v_{j}\right)-k_{1}\left(p_{i}-p_{n}\right)-k_{2}\left(v_{i}-v_{n}\right)
$$

where

(1) $-\sum_{j \in N_{i}} \nabla_{p_{i j}} V_{i j}$ is a gradient-based term consisting of a long-range attraction and short-range repulsion artificial potential force that achieves cohesion and separation. In particular, it is given in the following form:

$$
V_{i j}= \begin{cases}A\left(\frac{B^{2}}{\left\|p_{i j}\right\|}+\log \left\|p_{i j}\right\|^{2}\right), & \left\|p_{i j}\right\| \leq R \\ V_{R}, & \left\|p_{i j}\right\|>R\end{cases}
$$

where $p_{i j}=\left\|p_{i}-p_{j}\right\|$ represents the relative distance between agent $i$ and $j, A$ and $B$ are the regulating parameters of artificial potential field.

(2) $-\sum_{j \in N_{i}} c_{i j}\left(v_{i}-v_{j}\right)$ is the velocity consensus term that is responsible for velocity matching and $c_{i j}$ is the coupling strength regulating the weighted consensus value of velocity.

(3) $-k_{1}\left(p_{i}-p_{n}\right)-k_{2}\left(v_{i}-v_{n}\right)$ is the navigational feedback term that guides the motion of the whole flock, where $k_{1}, k_{2}>0$ are the navigational feedback gain, $p_{n}$ and $v_{n}$ are the position and velocity vector of the group objective or a virtual leader.

Utilizing the flocking control law (11), agents with arbitrary initial position and velocity distributions will form a cohesive formation and move along the predefined trajectory of the virtual leader. 


\subsection{Simulation Results and Discussion}

Based on the flocking control law (11), two simulation cases are performed with 10 agents moving in the plane. The initial positions of agents are randomly distributed within a $[0,20] \times[0,20] \mathrm{m}^{2}$ square area, the initial velocities are set with arbitrary directions and their magnitudes are within the range of $[0,10] \mathrm{m} / \mathrm{s}$ and the initial neighboring graph is supposed to be connected. The Simulation lasts for $15 \mathrm{~s}$ and the simulation step is chosen as $\Delta t=0.001 \mathrm{~s}$.

\section{Case I: Flocking with fixed coupling strength}

In this case, the coupling strength between agents is assumed to be fixed, i.e., if two agents are connected, $c_{i j}=1$; otherwise, $c_{i j}=0$. The simulation parameters are chosen as: $R=10, A=10, B=2, k_{1}=k_{2}=1$, the navigational velocity feedback $v_{n}=[5,0]^{\mathrm{T}} \mathrm{m} / \mathrm{s}$ and $p_{n}(0)=[20,10]^{\mathrm{T}} \mathrm{m}$ is the initial position of the virtual leader which updates according to (1).

Simulation results are shown in Figure 2, where Figure 2 (a) is the moving trajectories of all the agents, the circle ' $\circ$ ' represents each agent, solid line '-' denotes the trajectory of each agent; Figure 2 (b) shows the heading curves of all the agents and Figure 2 (c) demonstrates the variations of the coupling strength $c_{12}, c_{23}, c_{34}, \ldots, c_{910}$ between 9 agents.

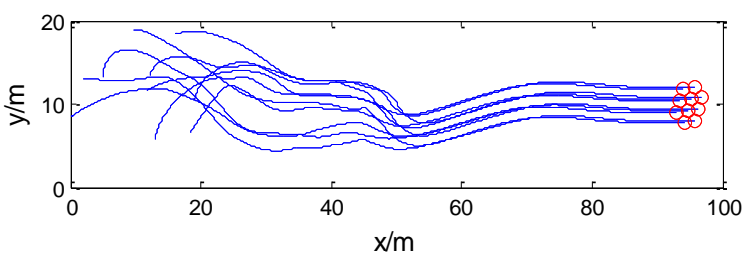

(a) Trajectories of all the agents

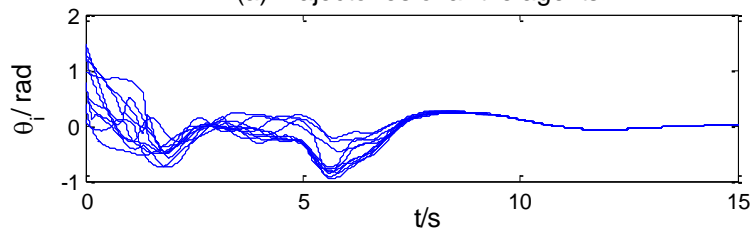

(b) Headings of all the agents

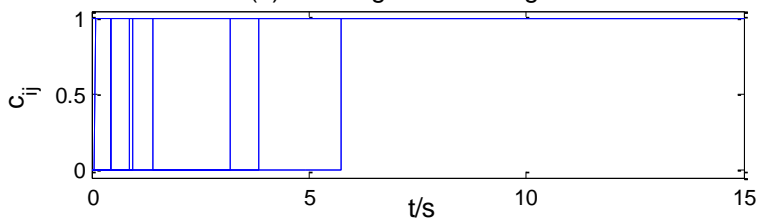

(c) Coupling strength of 9 agents

\section{Figure 2. Flocking With Fixed Coupling Strength}

\section{Case II: Flocking with varying coupling strength}

In this case, we utilize varying coupling strength which updates according to equation (10) during the simulation. Simulation parameters are chosen as: $r_{i j}=\mu_{i j}=\kappa_{i j}=1, h=0.2$, $\beta=1$, the other parameters are the same as Case I. Simulation results are shown in Figure 3 and the meaning of each sub-figure can be referred to Figure 2. 


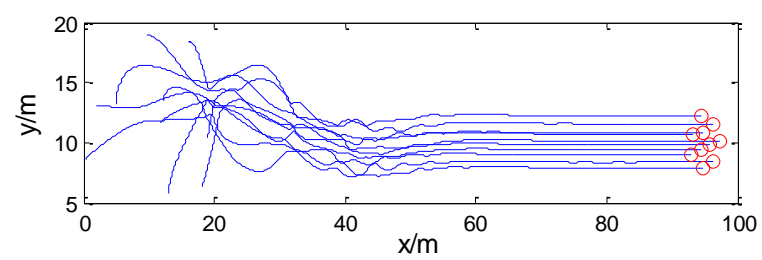

(a) Trajectories of all the agents

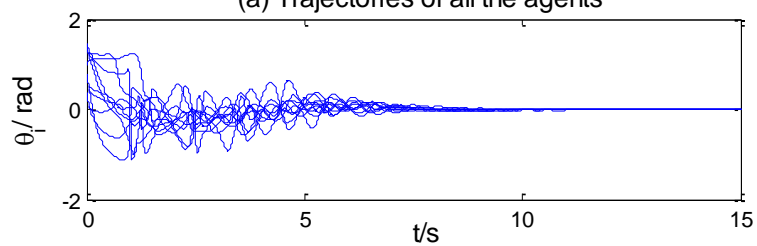

(b) Headings of all the agents

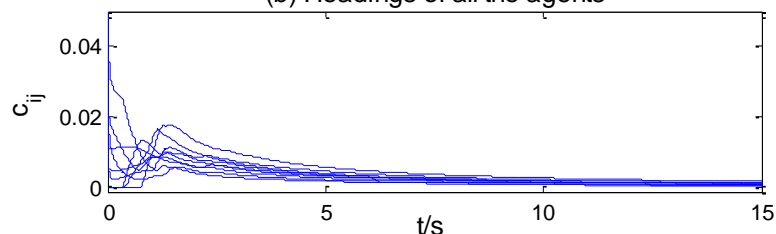

(c) Coupling strength of 9 agents

Figure 3. Flocking With Varying Coupling Strength

Discussion: Figure 2 and Figure 3 both demonstrate the moving trajectory, heading and coupling strength between 9 individuals in the two simulation cases. From which it can be seen that all agents can form a cohesive whole and move in formation, their headings converge to zero and the coupling strength between individuals converges to a fixed value eventually.

However, there are still some differences to be discussed in these two cases. Here, we analyze the results from three aspects:

(1) Moving trajectory: Figure 2 (a) and Figure 3 (a) provide the trajectories of all the agents during the simulation process, from which it can easily be seen that agents in Figure 3 (a) exhibit better flocking performance in the convergence to a steady state than that in Figure 2 (a). Individuals form a cohesive whole at about 50m in $x$ axis in Figure 3 (a), while in Figure 2 (a) individuals take a longer moving distance at about $80 \mathrm{~m}$ in $x$ axis to get into a steady state.

(2) Heading: Figure 2 (b) and Figure 3 (b) give the heading evolution of all the agents, from which we can see that the convergence time of all the headings in Figure 3 (b) is about $7 \mathrm{~s}$, while the results in Figure 2 (b) is about 13s, demonstrating that the convergence rate with varying coupling strength proposed in equation (10) is much faster than that with 0/1 fixed coupling strength, although the fluctuation of the curves in Figure 3 (b) is a little bigger than that of Figure 2 (b).

(3) Coupling strength: In Figure 2 (c), the coupling strength $c_{i j}$ hops from 0 to 1 discontinuously, only reflecting the connectivity of individuals. While in Figure 3 (c), coupling strength varies in a continuous mode and converges to a finite fixed value when the flock comes into a steady state. Note also that the coupling strength in Figure 3 (c) is much smaller (less than 0.05) than 1 during the evolution of the flocking process, which means that the optimal interaction intensity between agents can be a relatively small value rather than 1 .

From the simulation results and comparative analysis, we can conclude that the introduction of coupling strength in multi-agent system better reveals the internal interaction mechanisms of individuals and improves the flocking performance in time scale. 


\subsection{Extensions and Further Discussion}

To further analyze the internal interaction mechanisms between individuals in multiagent system, extended simulations are provided to illustrate the effect of different coupling factors on flocking. By adjusting the values of the coefficients of coupling strength $r_{i j}, \mu_{i j}$ and $\kappa_{i j}$, three comparative cases are studied.

As the heading of the virtual leader is 0 in this paper, here, we take the average heading $\Theta$ as the indicator for the flocking performance, which is defined as

$$
\Theta=\frac{1}{N} \sum_{i=1}^{N}\left|\theta_{i}\right|
$$

where $N$ is the number of agents, $\theta_{i}$ is the heading of the $i$ th agent. Obviously, $\Theta \in\left[\begin{array}{ll}0 & 2 \pi\end{array}\right]$ represents the heading convergence property of the flock. When $\Theta \rightarrow 0$, the heading of each agent in the flock converges to that of the virtual leader.

In order to investigate the effect of $r_{i j}$ on the flocking performance, we first let $\mu_{i j}=1$, $\kappa_{i j}=1$ during the simulation and choose $r_{i j}=1,3,5,8,10$, respectively. Five simulations are carried out and the time evolution of $\Theta$ can be obtained in Figure 4.

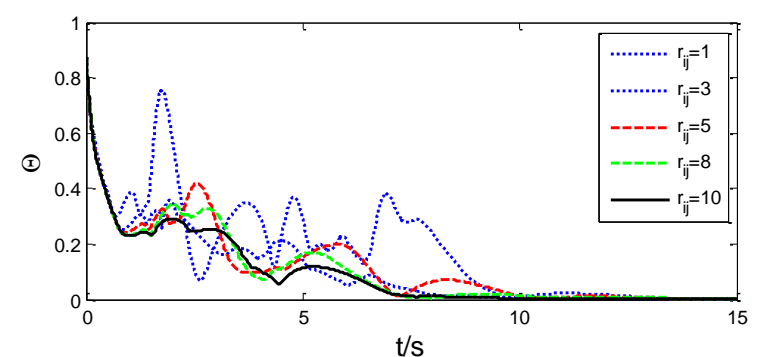

Figure 4. Comparative Results of $\Theta$ With Different $r_{i j}$

It is observed from Figure 4 that with the increase of $r_{i j}$, the rate of convergence of $\Theta$ also accelerates. Meanwhile, the fluctuation of $\Theta$ declines, demonstrating that bigger $r_{i j}$ is helpful in the convergence of the average heading $\Theta$.

Then, we set $r_{i j}=1, \kappa_{i j}=1$ and explore the relationship between $\Theta$ and $\mu_{i j}$. By varying the value of $\mu_{i j}$ as $\mu_{i j}=0.01,0.005,0.001,0.0005,0.0001$, the time evolution of $\Theta$ with different $\mu_{i j}$ is shown in Figure 5.

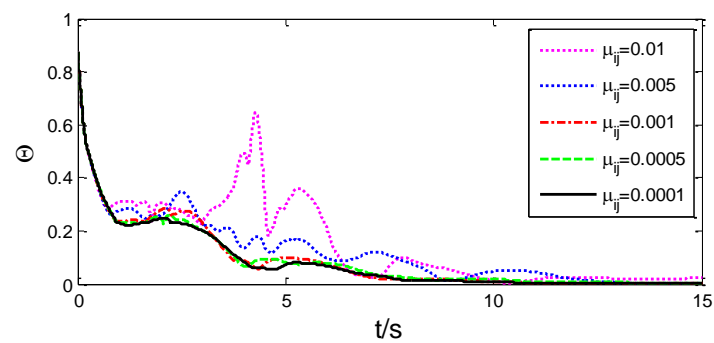

Figure 5. Comparative Results of $\Theta$ With Different $\mu_{i j}$

From Figure 5, we can see that as $\mu_{i j}$ decreases, the fluctuation of $\Theta$ declines and the curve of convergence becomes smoother, which also demonstrates that better flocking performance can be obtained when $\mu_{i j}$ is chosen to be a relative small value. 
Finally, we let $r_{i j}=1, \mu_{i j}=1$ and choose different $\kappa_{i j}$ as $\kappa_{i j}=3,1,0.5,0.1,0.05$, the effect of different $\kappa_{i j}$ on $\Theta$ is shown in Figure 6 .

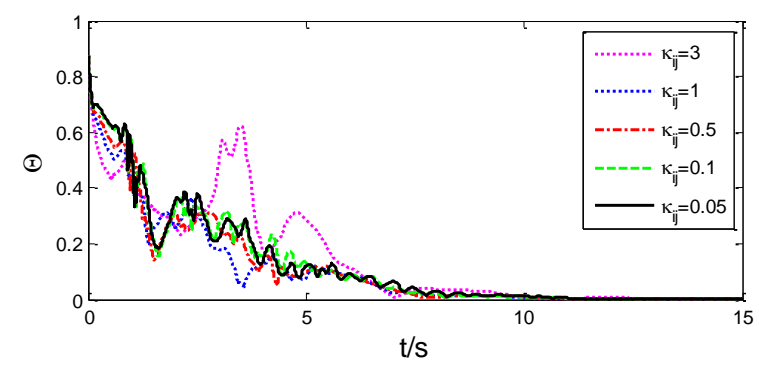

Figure 6. Comparative Results of $\Theta$ With Different $\kappa_{i j}$

As is demonstrated in Figure 6, the converge rate of $\Theta$ increases with the reduction of $\kappa_{i j}$ and the fluctuation of $\Theta$ also decreases.

From the three comparative analysis, we can conclude that the flocking performance of the whole group is relevant to the coupling factors explored in Section 2 and performance would be improved by choosing the coefficients of coupling strength $r_{i j}, \mu_{i j}$ and $\kappa_{i j}$ properly.

Moreover, note that the value of $\mu_{i j}$ chosen in Figure 5 is much smaller than that of $r_{i j}$, $\kappa_{i j}$ in Figure 4 and Figure 6, it means that the average headings $\Theta$ is more sensitive to velocity coupling factor than the other two factors. Research on this topic will be done in the future and is expected to find the dominant factor of coupling strength in the flocking process of multi-agent system.

\section{Conclusions}

In order to enhance the understanding of the internal interaction mechanisms in multiagent system, coupling strength between agents is investigated in this paper. Three factors relevant to the coupling strength are introduced and the detailed form of each factor is formulated. After that, comparative simulation studies between fixed coupling and varying coupling strength is performed under the same simulation condition, which demonstrates that the flocking performance can be enhanced with the proposed coupling strength. In addition, the influences of different factors on the flocking performance are investigated separately and the principles are obtained by comparative simulations. The result of this paper is expected to provide further insight into the underlying interaction mechanisms between individuals of multi-agent system.

\section{Acknowledgements}

This work is supported by the National Natural Science Foundation of China under Grants 51179156 and 51379176. 


\section{References}

[1] Y. Cao, W. Yu and W. Ren,“An overview of recent progress in the study of distributed multi-agent coordination”, IEEE Transactions on Industrial Informatics, vol. 9, no. 1, (2013), pp. 427-438.

[2] V. Gazi and K. M. Passino, "A class of attraction/repulsion functions for stable swarming aggregations", International Journal of Control, vol. 77, no. 18, (2004), pp. 1567-1579.

[3] W. Ren, "On consensus algorithmsfor double-integrator dynamics", IEEE Transactions on Automatic Control, vol. 53, no. 6, (2008), pp. 1503-1509.

[4] R. Olfati-Saber, "Flocking for multi-agent dynamic systems: algorithms and theory", IEEE Transactions on Automatic Control, vol. 51, no. 3, (2006), pp. 401-420.

[5] R. Olfati-Saber, "Consensus and cooperation in networked multi-agent systems", Proceedings of the IEEE, vol. 95, no. 1, (2007), pp. 215-233.

[6] W. Ren and R. W. Beard, "Distributed Consensus in Multi-Vehicle Cooperative Control: Theory and Application", London, Springer-Verlag, (2008).

[7] I. D. Couzin, J. Krause and N. R. Franks, "Effective leadership and decision making in animal groups on the move", Nature, vol. 433, no. 3, (2015), pp. 513-516.

[8] R. Lukeman, Y. Li and L. Edelstein-Keshet, "Inferring individuals rules from collective behavior", PNAS, vol. 107, no. 28, (2010), pp. 12576-12580.

[9] A. Eriksson, M. N. Jacobi and J. Nyström, "Determining interaction rules in animal swarms", Behavioral Ecology, vol. 21, (2010), pp. 1106-1111.

[10] Y. Katz, K. Tunstrøm and C. C. Ioannou, "Inferring the structure and dynamics of interactions on schooling fish", PNAS, vol. 108, no. 46, (2011), pp. 18720-18725.

[11] J. E. Herbert-Read, A. Perna and R. P. Mann,"Inferring the rules of interaction of shoaling fish", PNAS, vol. 108, no. 46, (2011), pp. 18726-18731.

[12] C. W. Reynolds, "Flocks, herds, and schools: a distributed behavioral model", Computer Graphics, vol. 21, no. 4, (1987), pp. 25-34.

[13] H. Su, X. Wang Z. Lin, "Flocking of multi-agents with a virtual leader", IEEE Transactions on Automatic and Control, vol. 54, no. 2, (2009), pp. 293-307.

[14] H. Shi, L. Wang and T. Chu, "Virtual leader approach to coordinated control of multiple mobile agents with asymmetric interactions", Physica D, vol. 213, (2006), pp. 51-65.

[15] T. Chu, L. Wang and T. Chen, "Self-organized motion in anisotropic swarms", Journal of Control Theory and Applications, vol. 1, no. 1, (2003), pp. 77-81.

[16] B. Liu, T. Chu and L. Wang, "Collective behavior analysis of a class of social foraging swarms", Proceedings of the 8th European Conference on Advances in Artificial Life, Canterbury, UK, (2005), pp. 584-593.

[17] S. H. Lee, "Predator's attack-induced phase-like transition in prey flock", Physics Letters A, vol. 357, (2006), pp. 270-274.

[18] J. Li and H. A. Sayed, "Modeling bee swarming behavior through diffusion adaption with asymmetric information sharing", EURSIP Journal on Advances in Signal Processing, vol.18, (2012), pp. 1-18.

[19] I. L. Bajec and F. H. Heppner, "Organized flight in birds", Animal Behavior, vol.78, (2009), pp. 777789.

[20] K. H. Charlotte and H. Hanno, "Some causes of variable shape of flocks of birds", PLoS One, vol. 6, no. 8, (2011), pp. 1-13.

[21] D. J. T. Sumpter, "The principles of collective animal behavior", Philosophical Transactions of the Royal Society B, vol. 361, (2006), pp. 5-22.

[22] N. W. F. Bode, D. W. Franks and J. A. Wood, "Limited interactions in flocks: relating model simulations to empirical data", Journal of the Royal Society Interface, vol. 8, (2011), pp. 301-304. 